\title{
Application of Edge Computing and SCADA System in Second Class Substation
}

\author{
Zhenyu Gao ${ }^{1}$, Pan Chen ${ }^{1}$, Zhonghua Chen ${ }^{1}$, Guochao Shi, Zhoncai Liu ${ }^{2 *}$, Shiwei Feng ${ }^{2}$ \\ ${ }^{1}$ Hangzhou Electric Power Design Institute Co., Ltd, Hangzhou, China \\ ${ }^{2}$ Shanghai Makesens EnergyStorage Technology Co.,Ltd, Shanghai, China
}

\begin{abstract}
This article adopts the way of progressive transformation of edge calculation and the combination of the substation, this paper makes a brief introduction to the calculation of edge, and describes its development situation at home and abroad, and then stand for intelligent SCADA system on $750 \mathrm{kV}$ substation briefly, the architecture will eventually margin calculation and combination of SCADA system, discuss the practicability of EC - SCADA system.
\end{abstract}

\section{Introduce}

With the increasing demand of electric load in all parts of the country year by year, the contradiction between China load Centre and primary energy centre is becoming more and more obvious, and the optimal allocation of energy resources in China is gradually paid attention to. In the current transmission mode of power system, UHV transmission reduces the engineering cost of unit power transmission with large transmission capacity and long transmission distance, reduces line loss, and saves the area of line corridor ${ }^{[1]}$. Improving comprehensive economic and social benefits plays an increasingly important role. As an important part of the intelligent substation of the second-class power transmission system, the SCADA (supervisory control and data acquisition) control and data acquisition system are responsible for many important uses, such as telecontrol function, automatic control function, meter function, interface and system use, relay protection and related functions. Its operation state can directly endanger the safe and stable operation of the power grid system.

\section{Edge Computing}

\subsection{Edge Computing Brief}

Edge computing refers to a new network architecture and open platform that integrates network, computing, storage and application of core capabilities at the edge of the network near the source of human, object or data acquisition.EC, the traditional centralized cloud computing processing mode is changed to move the storage computing power down to the edge of the network, that is the terminal oriented mode, which provides the edge intelligent service nearby. The edge computing mode greatly reduces the burden and blockage of the network caused by data return, realizes the relatively high transmission speed with low delay, and can quickly respond to the terminal request and improve the system performance. Figure 1 is a schematic diagram of cloud computing and edge computing.

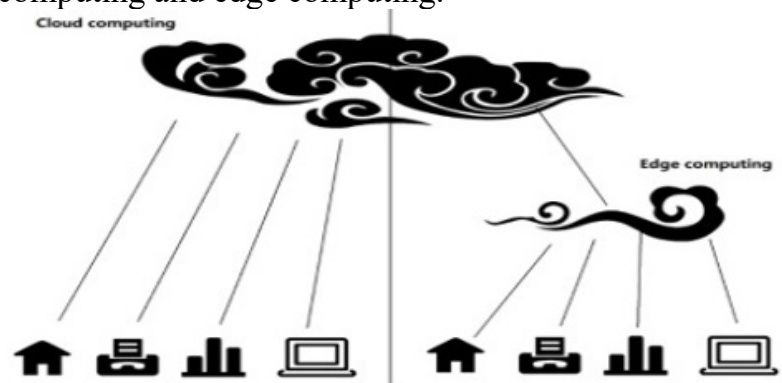

Fig.1. Comparison diagram between cloud computing and edge computing.

EC concept comes from $5 \mathrm{G}$ technology based on EC technology can realize the mutual operation function of different manufacturers and platform systems. The concept of EC can be extended to the Internet of things, and some experts are exploring EC application prospects in service manufacturing and other fields ${ }^{[2]}$.

The previous network cloud computing model is based on centralized core nodes. With the rapid development of intelligent substation, the research and development of various online monitoring systems, the number of process layer devices and systems is increasing day by day. Higher requirements are given for intelligent substation centralized data processing mode. For economic reasons, in order to prevent the centralization node from becoming a potential risk point and inhibit the development factor, the EC adopts a closer mode to the terminal side to respond to the data at the edge of the network, which not only improves the response speed of the system, but also reduce the system transmission requirements.

\footnotetext{
${ }^{*}$ Corresponding author: liuzhongcai0@ $163 . c o m$
} 


\subsection{Edge Computing Domestic and Foreign Development}

The edge computing is still in its early stages, Amazon, Google and Microsoft are still in the leading position in the edge cloud computing world, Amazon and AWS Greengrass jointly developed the edge computing field in 2017, and Microsoft plans to invest $\$ 5$ billion in emerging technology research in the next four years. Google also released two new products in 2017, hardware chip Edge TPU and software stack Cloud loT Edge ${ }^{[3]}$. Google said it Cloud loT Edg extend the powerful data processing and machine learning capabilities of Google Cloud to billions of edge devices.

Huawei Technology Co., Ltd., Shenyang Institute of Automation, Chinese Academy of Sciences, China Information and Communication Research Institute, Intel Corporation, ARM and Soft-com Power Information Technology Co., Ltd. jointly established in Beijing Edge Computing Consortium (ECC) ${ }^{[4]}$. A total of 62 members, covering scientific research institutions, industrial manufacturing, energy and electricity and other different fields. Version 1.0 and 2.0 of the Edge Computing Reference Architecture were published in 2016 and 2017, respectively, combing the test bed of edge computing, the solutions of edge computing in industrial manufacturing, power energy, smart city, transportation and other industries are proposed. Edge computing is one of $\mathrm{G}$ five core functions, Is the key to achieve $5 \mathrm{G}$ performance improvement. China Communication Standardization Association (CCSA) launched the Edge Computing Research Project in 2017. The CCSA Wireless Communications Commission (TC5) and the Industrial Internet Task Force (ST8) have established projects on edge computing, respectively. The three major operators have carried out extensive exploration in computing edge computing. In 2018, China Unicom announced the official launch of Edge-Cloud scale pilot projects and planning and construction of thousands of edge data centers in 15 provinces and cities across the country; China Mobile diverted to $\mathrm{CDN}$ edge nodes through core sinking gateways in Jiangsu and Zhejiang, and explored some commercial scenarios.

\section{Intelligent Station SCADA System Introduction and Architecture Model}

\subsection{SCADA System presentation}

SCADA system is a comprehensive application of modern automation, computer, signal processing and modern communication technology in the field of power substation, that is, the secondary equipment such as measurement and control, fault recording, protection and telecontrol device of substation are combined through function to realize automatic monitoring, measurement, control and information protection interaction of primary and secondary equipment of substation, as well as a series of comprehensive automation performance such as communication with dispatching center. SCADA system can complete the functions of monitoring, communication and information uploading by protecting, detecting, collecting, analyzing and calculating the information of the whole station.

\subsection{SCADA System Architecture Model in 750 kV Intelligent Substation}

For example, $750 \mathrm{kV}$ intelligent substation, SCADA system uses "Three-tier two-network [5]" hierarchical distributed architecture with 61850 protocol as the basic communication specification; "Three-tier" means the station control layer, the spacer layer and the process layer, it mainly includes backstage monitor, telecontrol, microcomputer five prevention, fault recording and other intelligent devices in the station; intelligent terminal, merging unit. The two networks are the station control layer network and the process layer network, corresponding to the MMS,GOOSE and SV network respectively. The three-tier two-net architecture is shown in Figure 2.

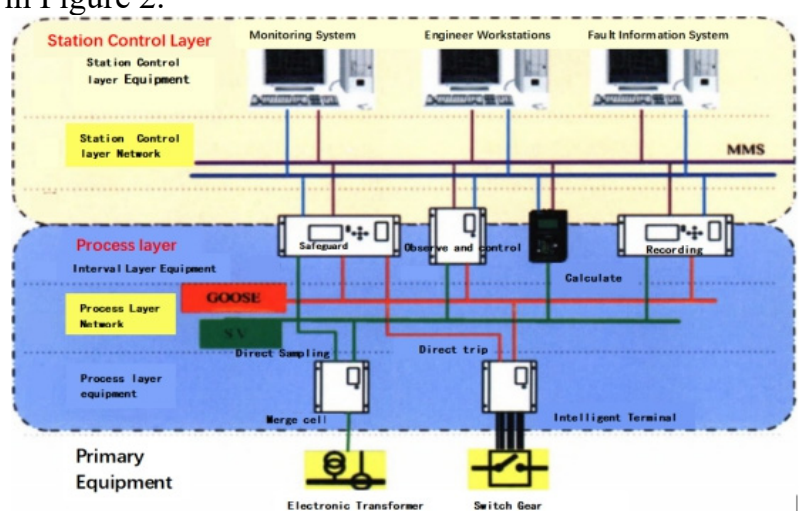

Fig.2. Three-tier two-network architecture.

\subsection{Failure analysis of SCADA system of intelligent station}

The normal and reliable operation of the intelligent station SCADA system is a necessary condition for the stable operation of the ultra-high voltage power transmission system. The normal and reliable operation of the SCADA system of the intelligent station not only depends on the functional design of the system itself, but is also closely related to the transmission channel, the status of the background server and the network status. Defects in any of these links may cause abnormalities in the entire intelligent station SCADA system. This section focuses on the shortcomings of the SCADA system of the smart station, namely communication interruption, server downtime and network storm.

\subsubsection{Communication interruption}

Communication interruption is one of the common factors of abnormal SCADA system of smart station. The communication transmission media of intelligent stations include optical cables, pigtail cables, jumpers and twistedpair cables. Small animal infringements, ground subsidence and excessive bending of man-made optical fibres are the main reasons for the damage of communication media. In addition to the communication 
interruption caused by the destruction of the communication medium and the destruction of the physical link, there are also communication interruptions caused by the terminal or background hardware and programs.

In general, communication interruption will not cause protection to directly affect the operation of primary equipment ${ }^{[6]}$. However, communication interruption can cause the background and dispatch centre to lose the remote information data such as the actual position of the primary equipment and the actual state of the secondary equipment, and the remote measurement data such as the current and voltage amplitude and phase, resulting in the loss of remote control of the primary and secondary equipment, and even The failure of automatic voltage and reactive power regulation (AVC) and other remote adjustments can endanger the reliable operation of the grid system in severe cases.

\subsubsection{Server down}

Due to the long running time, large amount of processed data and environmental factors, server downtime is also one of the reasons for the failure of the SCADA system of the smart station ${ }^{[7]}$.

On May 20, 2018, the operator of a $750 \mathrm{kV}$ substation found that the monitoring screen of the background monitoring machine No. 2 was black and could not start normally, and the background monitoring machine 2 server alarm light was always on. Initially, it is judged that the server program of the background monitoring machine 2 is dead, and the abnormality is still restored after restarting the server and the monitoring machine. After onsite inspection by maintenance personnel, it was judged that the hard disk of the background monitoring machine 2 server was damaged. After the hard disk was replaced, the system was restored to normal. It took about 19 hours from the discovery of the defect to the elimination of the defect.

Interactive output layer: Provide interactive data for the cloud background monitoring machine and dispatch centre, and provide man-machine dialogue.

The fast response layer is composed of a rule engine and a fast gateway, allowing core data to quickly communicate with the core processing layer and the interactive input layer.

Core processing layer data can be accessed by edge devices

The SCADA system server of the intelligent station is configured with double sets, and the operator station can be used as a monitoring machine. Therefore, restarting the server will generally not cause the station to lose monitoring.

\subsubsection{Network storm}

Due to the network topology and connection reasons, UDP packets are replicated on a large scale on the network and data frames are sent, which makes the network communication function drop sharply, and in severe cases, the network is completely paralyzed. The causes of network storms usually include: communication hardware reasons, equipment communication module abnormalities, network structure abnormalities and the use of hacker viruses ${ }^{[8]}$.

As one of the reasons for the failure of the SCADA system of the smart station, the network storm can lead to the overall failure of the SCADA system of the smart station, that is, the complete paralysis of the station control layer network.

\section{EC-SCADA System Application Inquiry}

EC-SCADA system can provide edge intelligent service nearby, realize the information exchange of automatic monitoring, measurement, control and protection of primary and secondary equipment of substation, and a series of comprehensive automation performance with dispatching center. According to the "three layers and two networks" architecture of intelligent substation ECSCADA the system architecture can realize the parallel calculation and analysis of various protection, measurement and control, stability control, measurement and accident record multiple data, so as to quickly draw the operation conclusion to meet the requirements of intelligent substation intelligent monitoring service.

\subsection{EC-SCADA System architecture}

EC-SCADA system architecture is shown in Figure 3, which is divided into four levels: interactive output layer, fast response layer, core processing layer and edge device interface layer. The interactive output layer provides interactive data for cloud background monitor and dispatch center, provides man-machine dialogue; fast response consists of rule engine and fast gateway to enable core data to communicate quickly with interactive output layer; core processing layer data can be integrated with terminal equipment, including core data center, metadata, registry and configuration ${ }^{[9]}$.

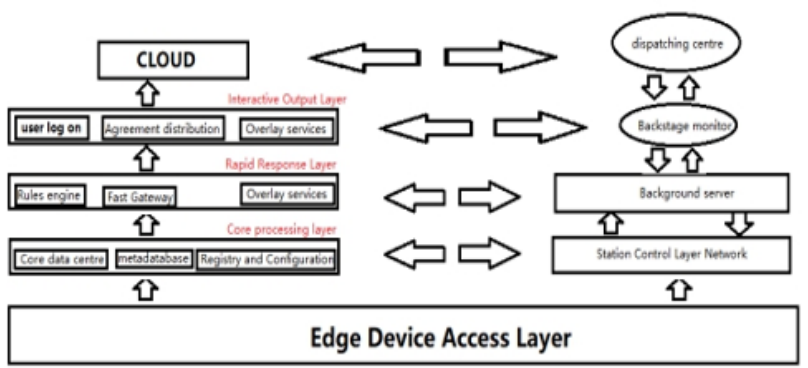

Fig.3. EC-SCADA system architecture.

\subsection{Comparison of EC -SCADA and SCADA Systems Scheduling Remote Control}

SCADA the remote control of the dispatching system, the remote control is first selected by the dispatching master station through telecontrol ${ }^{[10]}$. After success, the terminal equipment carries on the remote-control reverse calibration to the dispatching master station through the telecontrol. After receiving the Return Correction, the 
dispatching master station performs remote control to the terminal equipment ${ }^{[11]}$.

EC-SCADA system scheduling remote control, through the dispatching master station at the same time to the cloud and telecontrol remote control selection, due to EC-SCADA system characteristics, communication speed reasons, cloud command priority through the core processing layer sent to the terminal equipment to select, after success, the terminal equipment Return Correction, the core processing layer directly controls the terminal equipment after receiving the return ${ }^{[12]}$. EC-SCADA system diagram is shown in Figure 4.

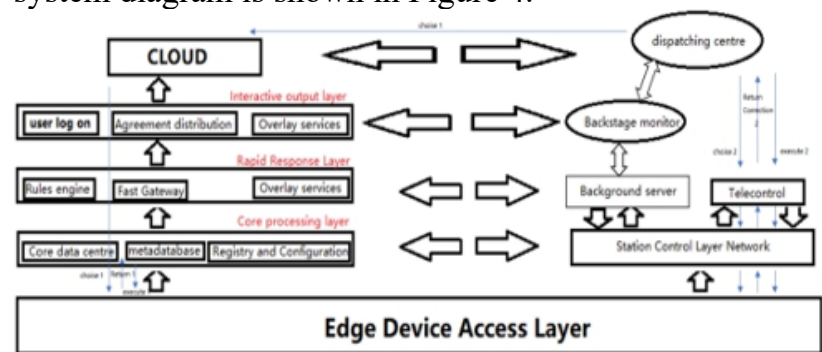

Fig.4. EC-SCADA system diagram.

Through simulation and comparison EC-SCADA the system performs faster and more reliably than the SCADA system. Meanwhile EC-SCADA the system can avoid the problem of partial function locking and system paralysis caused by SCADA system because of link selection.

\section{Significance of EC-SCADA systems}

EC-SCADA system improves the intelligence of the system, reduces the dependence of centralized data center, reduces the processing cost of data transmission, can complement each other with telecontrol and existing background monitoring server, and EC-SCADA the system to improve the terminal data processing and decision-making ability of intelligent substation. Moreover, the system improves the processing ability of heterogeneous data in remote monitoring disk of intelligent substation, and provides a reliable guarantee for the safe and stable operation of UHV transmission.

\section{Conclusion}

EC provide intelligent services in the network edge measurement fusion network, computing, storage and so on, especially suitable for the application of SCADA systems with a large number of different manufacturers and different principles of intelligent terminals. This paper has made the framework design of the EC-SCADA system for the existing situation, but there are still some key technical problems in practice. With the continuous development of the technology and the improvement of the relevant standards, under the background of the development of global energy interconnection EC knitting can promote the technological innovation of the next generation intelligent substation.

\section{References}

1. Huangzhang Lv, Chen Dan, Fan Bin, et al. Standardization progress and case analysis of edge computing (JCRD, Guangzhou, 2018)

2. Bin Li, Bincheng Jia, Wangzhang Cao, et al. Application prospect of edge computing in power demand response business (Phys. S.T, Shanghai, 2018)

3. Zhang Ning, Jingku Liu. The ultra-high voltage grid risk analysis and its assessment framework (Phys. S.P.C, Beijing, 2013).

4. Chao Chen, Shouwei Zhang, et al. Research on power industry data application based on big data. (MET, Shanghai, 2014).

5. Wang Xun, Zhao Meng, et al. Discussion on big data analysis and application of power transmission and transformation equipment (Phys.SBD, Shengzhen, 2018)

6. Ziming Zhao, Zhiping Cai, et al. Edge computing: plating, application and challenge (JCRD, Xi'an, 2018)

7. Yanzhen Lin, Qingwu Gong. Relay protection risk assessment based on fuzzy information granulation algorithm (EPC, Beijing, 2016)

8. Yue Yu, Chunxia Zhou, Rongrong Zhan, et al.reaserch on network stress cause and test method of relay protection in smart substation(PSPC, Changsha,2017)

9. Haitao Yang, Ping Ji, Yuan Ren, et al. Analysis of safety monitor function and technical requirements for UHV power grid (PST, Shanghai, 2016)

10. Miao Miao,Ping Ji et al. analysis on interrelationship between future UHV power grid structural form and power source composition in china (AEPS, Shanghai,2018)

11. Zhaoyang Xue, Yening Lai. Integration of macro energy thinking and big data thinking part one big data and power big data (AEPS, shanghai, 2016)

12. Zhaoyang Qu, Shuai Chen, Fan Yang, et al. An attribute reducing method for electric power system (AEPS, Shanghai, 2014) 